\title{
Pulmonary Adenosquamous Cell Carcinoma With Systemic Lymphadenopathy due to Immunoglobulin G4-Related Disease: A Case Report
}

\author{
Saori Ikebe ${ }^{\mathrm{a}}$, Seigo Minami ${ }^{\mathrm{a}, \text { b, d }}$, Shouichi Ihara ${ }^{\mathrm{a}, \mathrm{b}}$, Hironao Yasuoka ${ }^{\mathrm{c}}$, Kiyoshi Komuta ${ }^{\mathrm{b}}$
}

\begin{abstract}
A 75-year-old man with diabetes mellitus showed elevated C-reactive protein (CRP) level at his regular visit. Computed tomography scan showed a lung tumor in his left lower lobe and systemic lymphadenopathy including abdominal lymph nodes. The patient was diagnosed as primary pulmonary squamous cell carcinoma with systemic lymph node metastasis. Thereafter, unexpected steroid pulse therapy for accidental acute exacerbation of interstitial pneumonia rapidly shrank lymphadenopathy. At this time, we also found elevated serum immunoglobulin G4 (IgG4) level (385 mg/dL). Considering these findings, we doubted the lymph nodes metastases at the initial staging, and then corrected cancer-staging (C-staging) from inferior vena cava (IVC) to inferior abdomen (IA). In addition, during the steroid tapering, sudden onset and uncontrollable left pneumothorax required surgical approach. Curative-intent left lower lobectomy with lymphadenectomy was performed for the lung cancer. Pathological findings revealed coexistence of adenosquamous carcinoma and infiltration of IgG4-positive plasma cells in the resected mediastinal lymph node. We detected 384 IgG4-positive cells per high power field. IgG4/ IgG-positive cell ratio was $54 \%$. Based on these findings, the diagnosis of IgG4-related disease with primary adenosquamous carcinoma (p-stage IIIA) was confirmed. The patient died 24 days after surgery because of another acute exacerbation of interstitial pneumonia. Our case alerts oncologists to IgG4-related disease as a possible underlying comorbidity which may confuse pretreatment clinical stage.
\end{abstract}

Keywords: Adenosquamous cell carcinoma; IgG4-related disease; Interstitial pneumonia; Lymphadenopathy; Acute exacerbation; Fludeoxyglucose-positron emission tomography

Manuscript submitted January 11, 2021, accepted January 27, 2021

Published online February 8, 2021

aDepartment of Respiratory Medicine, Osaka Police Hospital, 10-31 Kitayama-cho, Tennoji-ku, Osaka 543-0035, Japan

bDepartment of Respiratory Medicine, Daini Osaka Police Hospital, 2-6-40 Karasuga-tuji, Tennoji-ku, Osaka 543-0042, Japan

'Department of Pathology, Osaka Police Hospital, 10-31 Kitayama-cho, Tennoji-ku, Osaka 543-0035, Japan

${ }^{\mathrm{d}}$ Corresponding Author: Seigo Minami, Department of Respiratory Medicine, Osaka Police Hospital, 10-31 Kitayama-cho, Tennoji-ku, Osaka-City, Osaka 543-0035, Japan. Email: seigominami@oph.gr.jp

doi: https://doi.org/10.14740/jmc3649

\section{Introduction}

Immunoglobulin G4 (IgG4)-related disease is a systemic syndrome characterized by tissue infiltration of small lymphocytes and IgG4-positive plasma cells, and following fibrosis. This disease affects pancreas, salivary glands, artery, thyroid, lung, and many other organs. It remains controversial whether IgG4-related disease is associated with the risk of malignancy or not. In some studies, malignancy increased in IgG4-related disease $[1,2]$ or only in autoimmune pancreatitis [3, 4]. On the contrary, a Japanese study could not find any difference in the incidence of total malignancies between 113 patients with IgG4-related disease and a general population [5].

Serum level of IgG4 elevates in most cases. Although the diagnosis is based on biopsy, it is sometimes difficult to obtain pathological specimen. The diagnostic criteria of IgG4-related disease are defined by the following three findings: clinical manifestations, elevated serum IgG4 level, and pathological characteristics. Clinical manifestations of IgG4related disease are similar to cancer-related manifestations in some cases. In a UK study of 53 patients with IgG4-related disease, 22 patients $(41.5 \%)$ were categorized as definite, highly probable or probable thoracic involvement [6]. The following four patterns of radiological manifestation have been described for IgG4-related lung disease: solid nodular, bronchovascular, alveolar interstitial, and round shaped ground-glass opacity type [7]. These patterns mimic various lung diseases, including lung cancer, interstitial pneumonia and sarcoidosis.

Herein, we report a case of co-existence of pulmonary adenosquamous cell carcinoma and IgG4-related disease manifested by systemic lymphadenopathy, which confused our clinical staging and treatment strategy.

\section{Case Report}

A 75-year-old man with diabetes mellitus showed elevated C-reactive protein (CRP) level at his regular visit. Computed tomography (CT) scan found a tumor in the left lower lobe, interstitial pneumonia (Fig. 1a), and systemic lymphadenopathy including peri-gastric and peri-pancreatic lymph nodes. Bronchoscopic biopsy pathologically diagnosed the lung tumor as a squamous cell carcinoma in the middle of July 2016. 

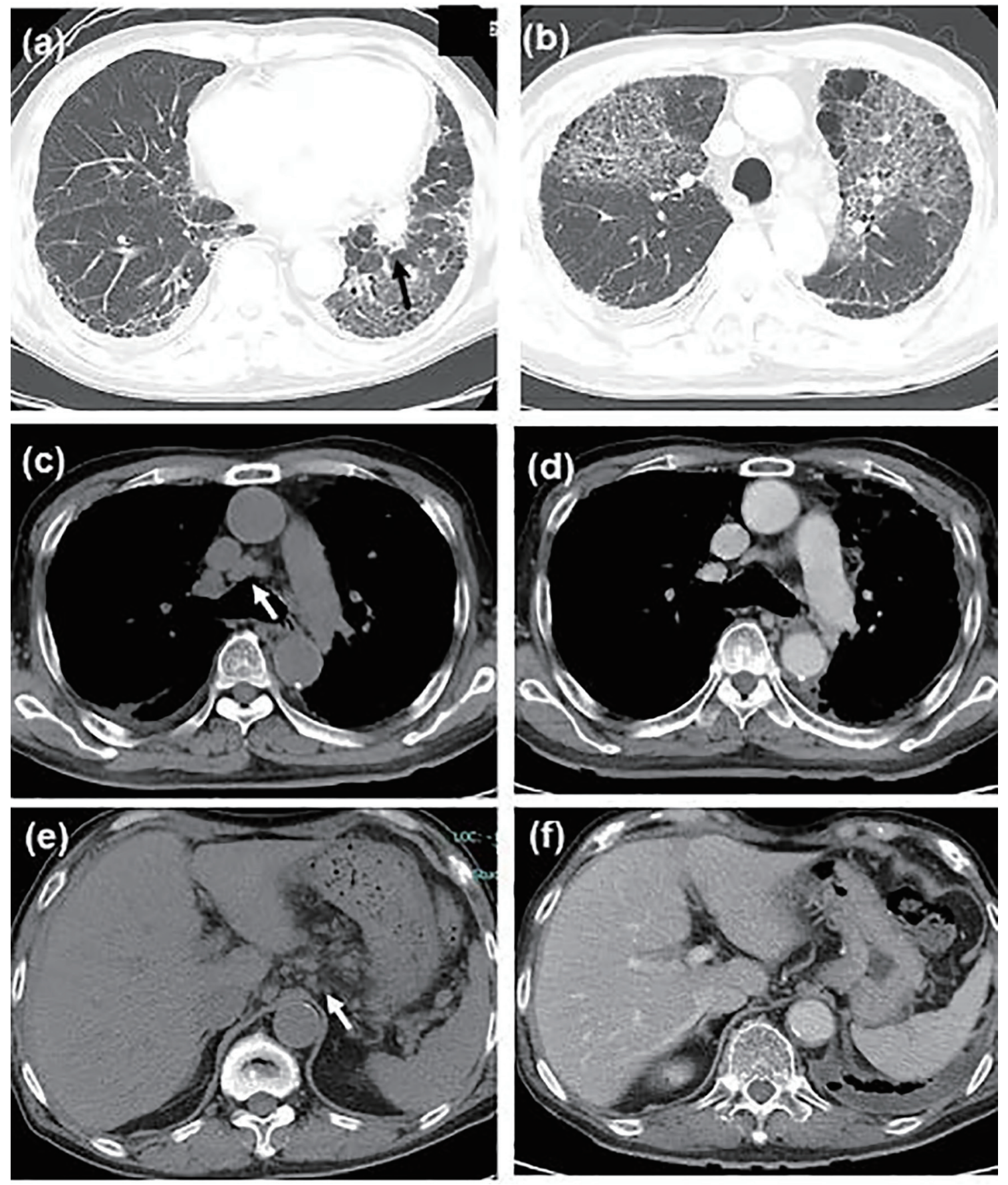

Figure 1. Chest computed tomography (CT) scan showing (a) the primary tumor in the left lower lobe (shown by black arrow) and bilateral sub-pleural reticular shadows in the bilateral lower lobes, (b) the first acute exacerbation of interstitial pneumonia, remarkable shrinkage of (c, d) mediastinal and (e, f) peri-gastric lymphadenopathy (c, e) before steroid therapy (shown by white arrows, in the middle of September) and ( $d, f)$ during steroid tapering (in the late October).

Esophagogastroduodenoscopy, endoscopic sonography, and contrast-enhanced magnetic resonance imaging (MRI) could not detect abdominal malignancy. Enhanced brain CT did not find brain metastasis. Fludeoxyglucose-positron emission tomography (FDG-PET) showed abnormal uptake in the lung tumor in the left lower lobe (maximum standardized uptake value (SUVmax), 4.7), and systemic lymph nodes in the supraclavicular fossa (SUVmax, 6.8), subaortic arch (SUVmax, 4.0), gastric cardia (SUVmax, 3.5), peri-pancreas (SUVmax, 4.3), superior mesenteric arterial root (SUVmax, 5.1) (Fig. 2). Considering these findings, we diagnosed the lymphadenopathy as metastasis of lung cancer, and then planned chemotherapy for 

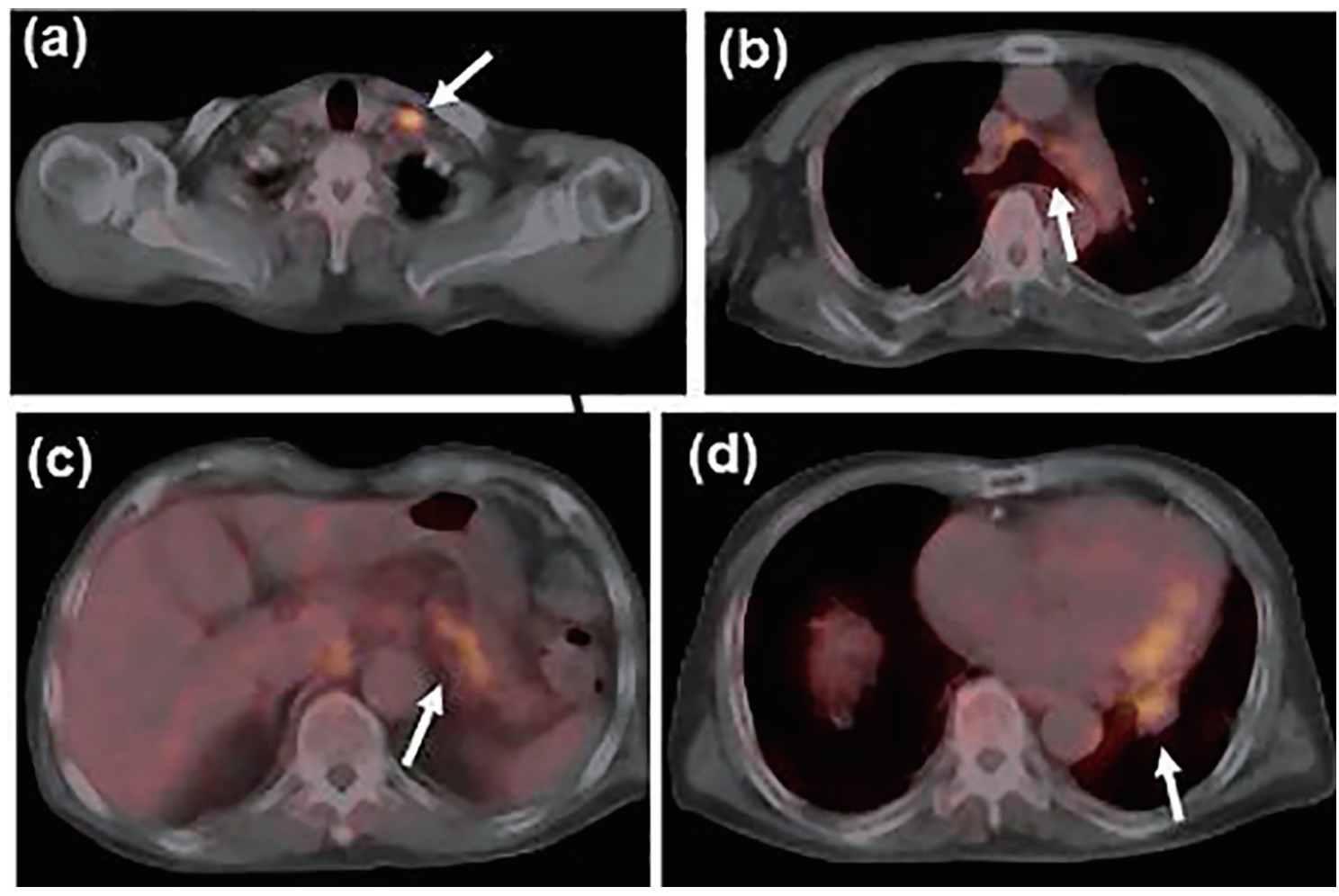

Figure 2. Fludeoxyglucose-positron emission tomography (FDG-PET) scan at the initial staging before steroid therapy showed uptake in (a) the left supraclavicular, (b) mediastinal and (c) peri-gastric lymph nodes, and (d) the primary tumor in the left lower lobe (shown by white arrows).

clinical stage IV cancer. However, acute exacerbation of interstitial pneumonia canceled the pre-planned chemotherapy in the middle of September (Fig. 1b). We immediately started intravenous pulse-steroid therapy at the dose of $1 \mathrm{~g}$ /day of methylprednisolone during the first consecutive 3 days, and thereafter gradually tapered steroid dose from $40 \mathrm{mg} /$ day of prednisolone. During the steroid tapering, left pneumothorax suddenly occurred. Aspiration from two chest drainage tubes failed to control air leakage. Thus, we planned surgical approach for uncontrollable pneumothorax. Another CT scan before surgery revealed remarkable shrinkage of systemic lymph nodes (Fig. $1 \mathrm{c}-\mathrm{f})$. We doubted the decision of lymph nodes metastases at the initial staging, and then corrected C-staging from C-stage inferior vena cava (IVC) to C-stage inferior abdomen (IA) $\left(\mathrm{cT}_{1 \mathrm{~b}} \mathrm{~N}_{0} \mathrm{M}_{0}\right)$. We also found elevated IgG4 level $(385 \mathrm{mg} / \mathrm{dL})$, KL-6 $(661 \mathrm{U} / \mathrm{mL})$ and SP-D $(407 \mathrm{ng} / \mathrm{mL})$ in the serum obtained before the steroid pulse therapy, and then decreased IgG4 level (193 mg/dL) and SP-D (67.6 ng/mL), but stable KL-6 (675 U/ $\mathrm{mL}$ ) in the serum obtained during the steroid tapering and just before the subsequent operation. There was no radiological indication of IgG4-related disease in other organs. Curativeintent left lower lobe lobectomy with lymphadenectomy was performed for the lung cancer at the end of October. The pneumothorax was successfully cured and did not recur thereafter. The resected left lower lobe contained mainly squamous cell carcinoma with keratinization (Fig. 3a), partial component of adenocarcinoma (Fig. 3b), and interstitial pneumonia (favor for non-specific interstitial pneumonia (NSIP)) with marked lym- phoplasmacytic cell infiltration into interstitium of interlobular septal walls (Fig. 3c, d). Both resected lung and mediastinal lymph nodes contained epithelioid granulomas with necrosis, suggesting complication of pulmonary tuberculosis (Fig. 3e, f). Thereafter, mycobacterium tuberculosis was found to be negative in smear, but positive in culture of post-operative sputum specimen. Combination of four anti-tuberculosis drugs (isoniazid, rifampicin, ethambutol and pyrazinamide) started 18 days after the surgery. The resected mediastinal lymph nodes contained not only malignant cells (Fig. 3f) but also IgG4-positive plasma cells (Fig. 4). We could not detect typical pathological findings of IgG4-related disease, such as obliterative phlebitis or storiform-fibrosis. Pathological staging was determined as $\mathrm{pT}_{2 \mathrm{a}} \mathrm{N}_{2} \mathrm{M}_{0}$. We detected 384 IgG4-positive cells per high power field, and IgG4/IgG-positive cell ratio was 54\% (Fig. 4). These findings confirmed the diagnosis of IgG4-related disease. Infiltration of IgG4-positive cells was also seen in lung tissue of diffuse ground-glass opacity, suggesting IgG4-related interstitial pneumonia. Another acute exacerbation of interstitial pneumonia occurred 8 days after surgery. Despite of steroid therapy, the patient died 24 days after surgery in late November.

\section{Discussion}

This was a rare case of co-existence of locally advanced pulmonary adenosquamous cell carcinoma and IgG4-related disease manifested by systemic lymphadenopathy. To our knowledge, 

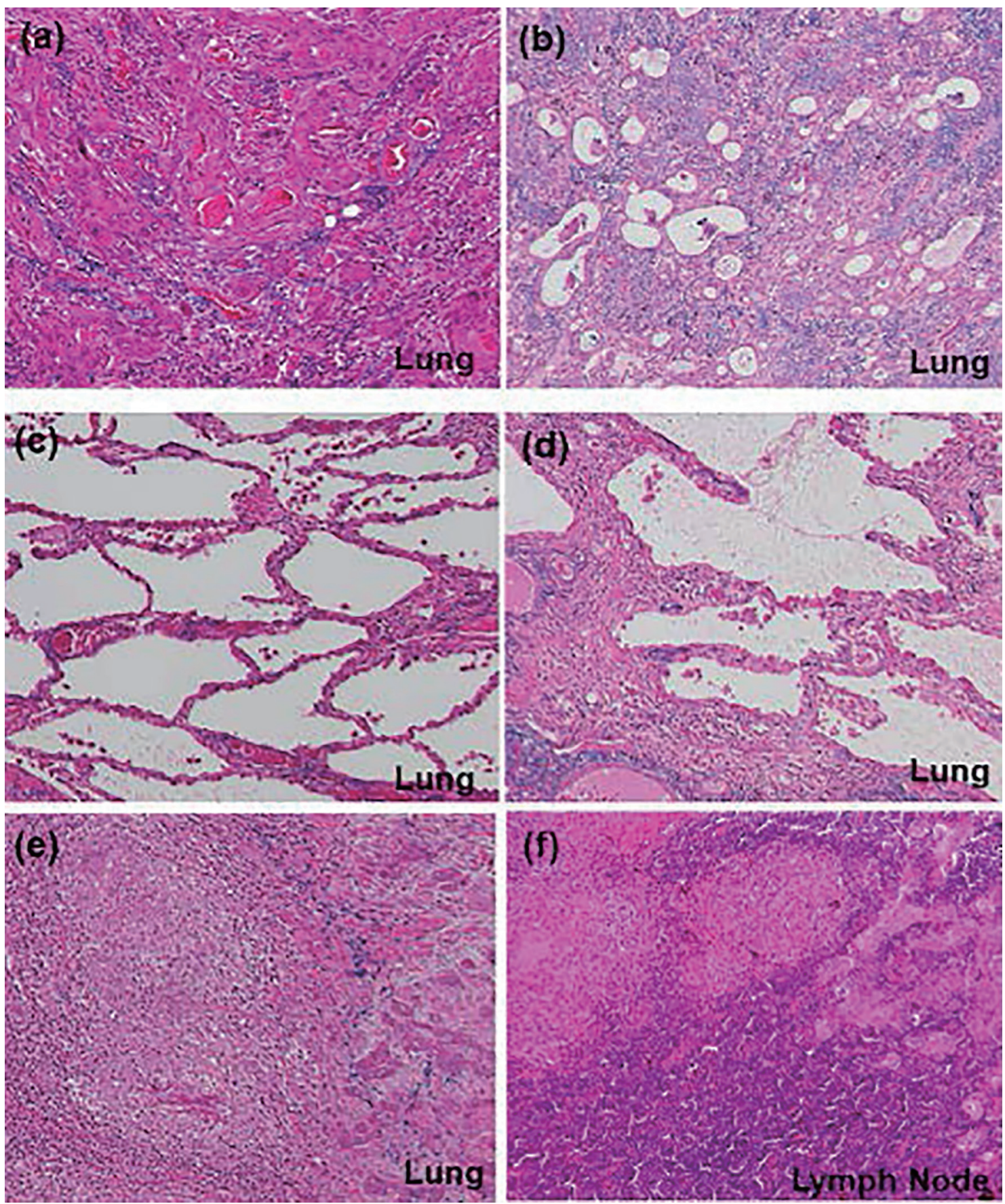

Figure 3. Histological findings (hematoxylin and eosin (H\&E) stain, $\times 10)$ of (a) main component of squamous cell carcinoma and (b) partial component of adenocarcinoma in the primary tumor, (c) interstitial pneumonia (favor for non-specific interstitial pneumonia), (d) lymphoplasmacytic cell infiltration into interstitium of interlobular septal walls, (e) coexistence of epithelioid granulomas and primary squamous cell carcinoma in the left lower lobe, and (f) coexistence of epithelioid granulomas and metastatic lesions in the mediastinal lymph nodes. (e, f) These findings suggest complication of pulmonary tuberculosis and lung cancer.

there were only nine case reports of lung cancer concomitant with IgG4-related disease [8-16] (Table 1).

A unifying diagnostic criterion has not been established, because various organs are involved in this disease. The current diagnosis of IgG4-related disease is based either on comprehensive or organ-specific diagnostic criteria. According to the com- prehensive diagnostic criteria for IgG4-related disease published in 2011 by the Japanese IgG4 team, organized by the Ministry of Health, Labor and Welfare (MHLW) of Japan [17], our case met the three diagnostic factors: chest imaging, serology and histology. Based on the eight weighted inclusion criteria domains, the third step of classification process, of the 2019 American Col- 

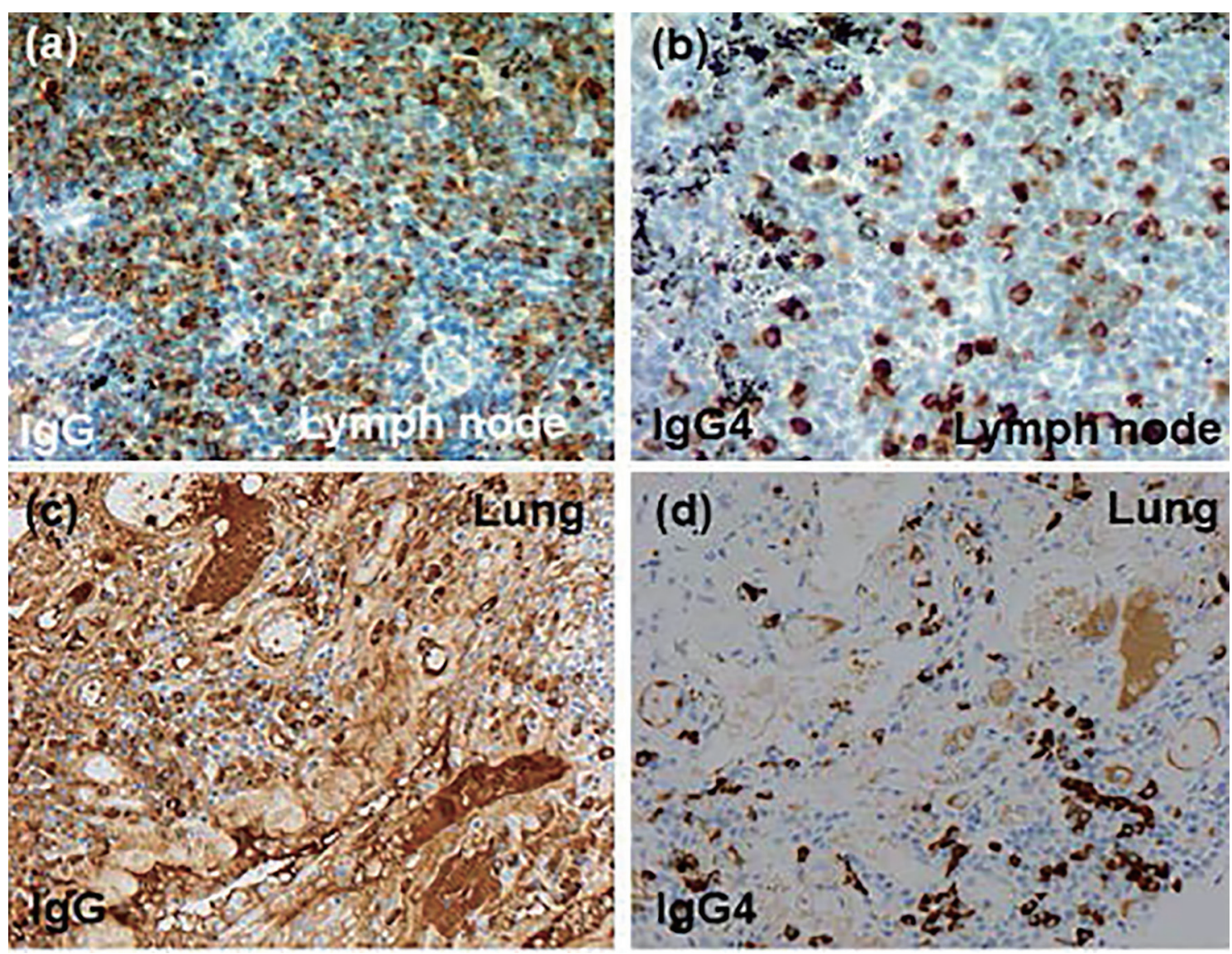

Figure 4. Immunohistochemical findings $(\times 20)$ : $(a, b)$ mediastinal lymph node, $(c, d)$ lung tissue, $(a, c)$ anti-lgG antibody and (b, d) anti-IgG4 antibody. IgG4: immunoglobulin G4.

lege of Rheumatology/European League against Rheumatism Classification Criteria for IgG4-related Disease [18], our case presented dense lymphocytic infiltrate ( +4 points), the $\operatorname{IgG} 4 /$ IgG ratio $41-70 \%$ and the number of IgG4-positive cells/high power field $>10$ cells ( +14 points), and $2-5 \times$ upper limit of normal serum IgG4 concentration ( +6 points). Thus, our case exceeded the threshold of 20 points. In light of the diagnostic criteria for IgG4-related respiratory disease proposed by Matsui et al in 2016 [19], our case met the chest imaging of hilar and mediastinal lymphadenopathy, the elevated serum IgG4 concentration, marked lymphoplasmacytic cell infiltration into interstitium of interlobular septal wall, and the IgG4/IgG-positive cell ratio $>40 \%$ and $>10$ IgG4-positive cells/high power field. Thus, our case was a possible diagnosis of IgG4-related respiratory disease. The latter two diagnostic criteria were published after our patient was already dead or at the same time of our surgery, respectively. Despite of satisfaction of these various inclusion criteria, our case did not meet the exclusion criteria due to pulmonary comorbidities of lung cancer and tuberculosis. A significant correlation between malignancy and IgG4-related disease has indicated IgG4-related disease as a paraneoplastic syndrome, rather than an independent disease [20]. Furthermore, we could not completely exclude other similar diseases, because we did not examine various serological markers suggestive of another systemic autoimmune disease, vasculitis or sarcoidosis such as antinuclear antibody, rheumatoid factor, and anti-neutrophil cytoplasmic antibody (ANCA). Thus, in the current and latest diagnostic criteria, our diagnosis was not enough for the strict definition of IgG4-related disease.

We thought it impossible to distinguish IgG4-related lymphadenopathy from lymph node metastases only by routine images of clinical staging. Either endobronchial ultrasoundguided transbronchial needle biopsy (EBUS-TBNB) or mediastinoscopical biopsy should be considered aggressively for confusing lymphadenopathy. In our case, neither pre-staging enhanced CT scan nor FDG-PET could detect the underlying IgG4-related disease. As a result, the remarkable shrinkage of lymph nodes after unexpected steroid therapy reminded us of IgG4-related disease. Surgical resection finally determined the IgG4-related systemic lymphadenopathy, and then downstaged pre-surgical clinical staging from IV to IIIA. In fact, most previous cases required surgical biopsy for the diagnosis of IgG4-related disease (Table 1). We oncologists have to keep in mind a malignancy mimicker, IgG4-related disease [21].

The second important finding was post-surgical acute exacerbation of IgG4-related lung disease, NSIP pattern. In a UK study, interstitial lung disease of NSIP pattern was observed in $22.7 \%$ of IgG4-related thoracic disease. In this study, among four dead patients with thoracic involvement, NSIP was considered to have contributed to the patient's death in two cases 


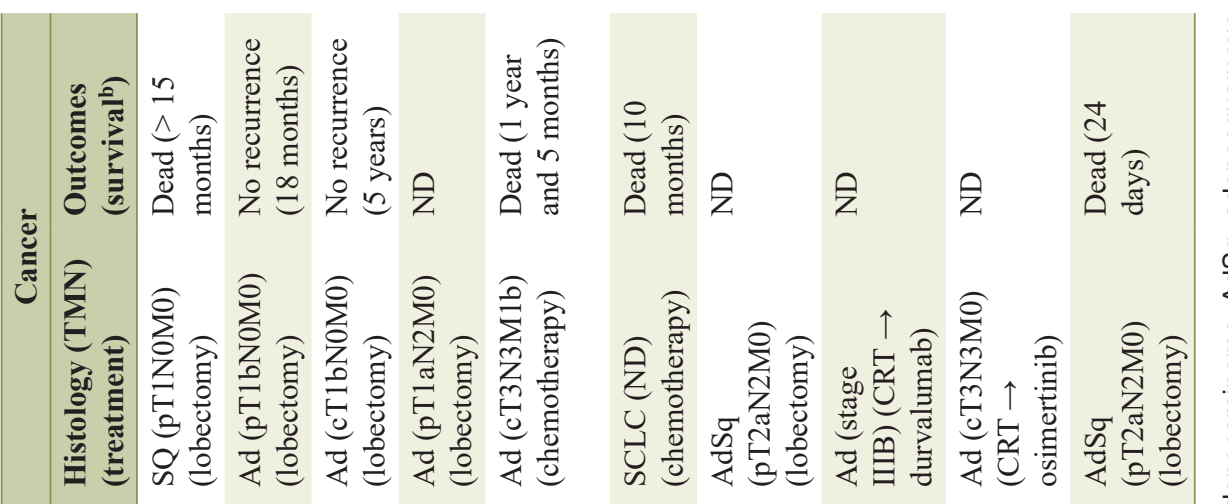

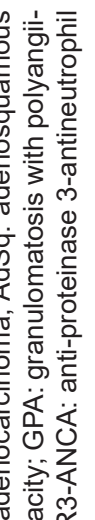

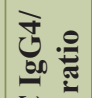

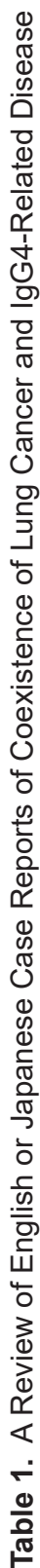

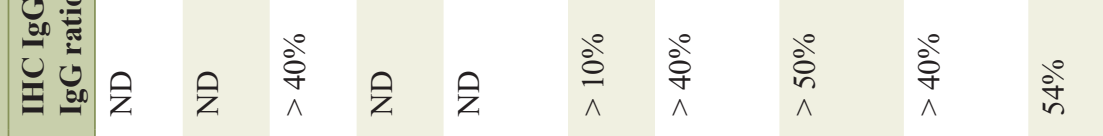

प्र

要

$\frac{3}{30} \approx$

कृ
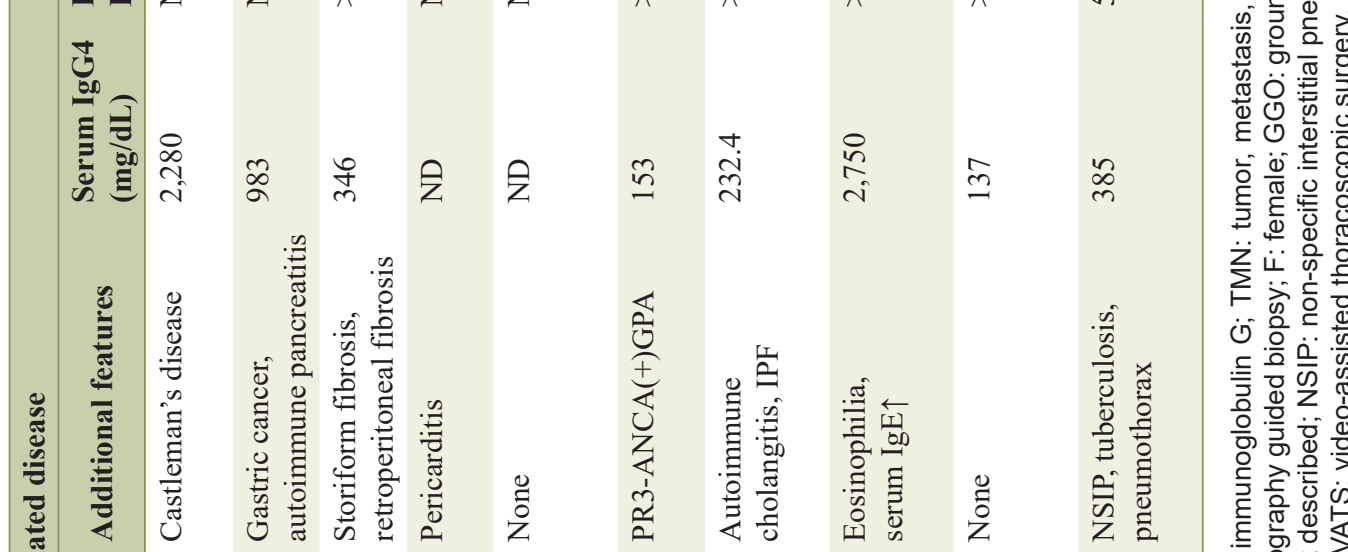

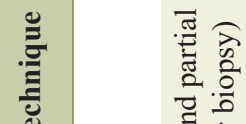

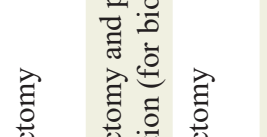

焉

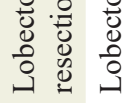

$\begin{array}{ll} & \overrightarrow{0} \\ \text { हे } & 0 \\ 0 & 0 \\ 0 & 0 \\ 0 & 0 \\ 0 & 0 \\ 0 & 0\end{array}$

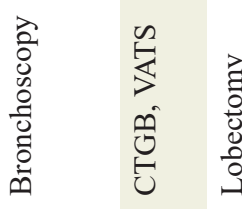

崖

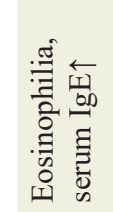

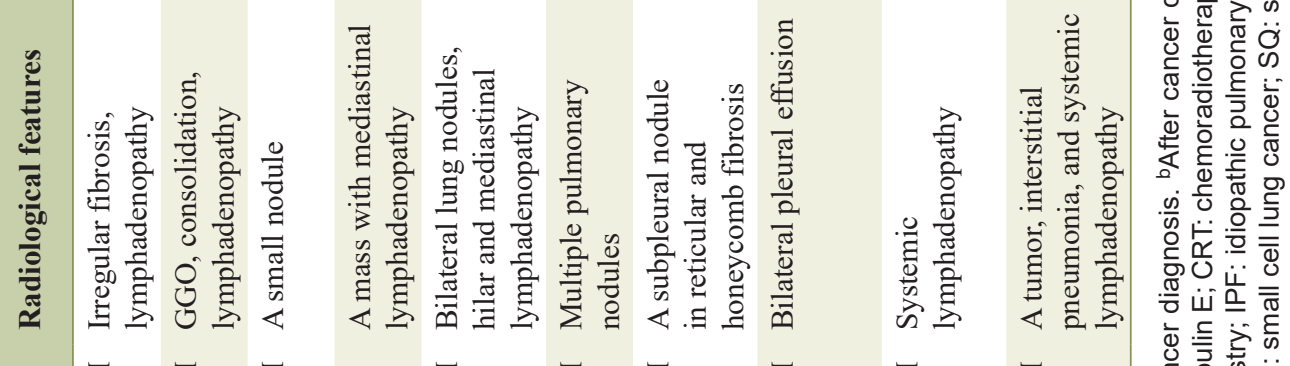

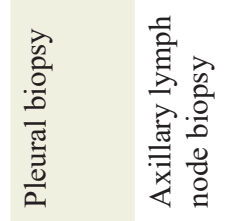

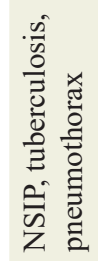

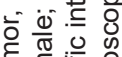

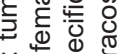

zั山े के

ô के o

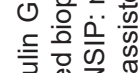

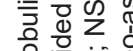

응 음 这

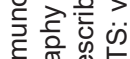

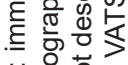

نั

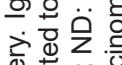

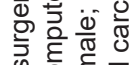

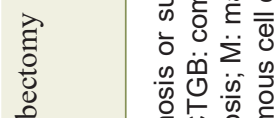

든 क

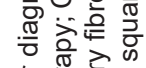

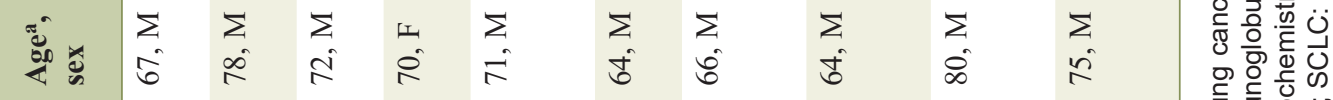

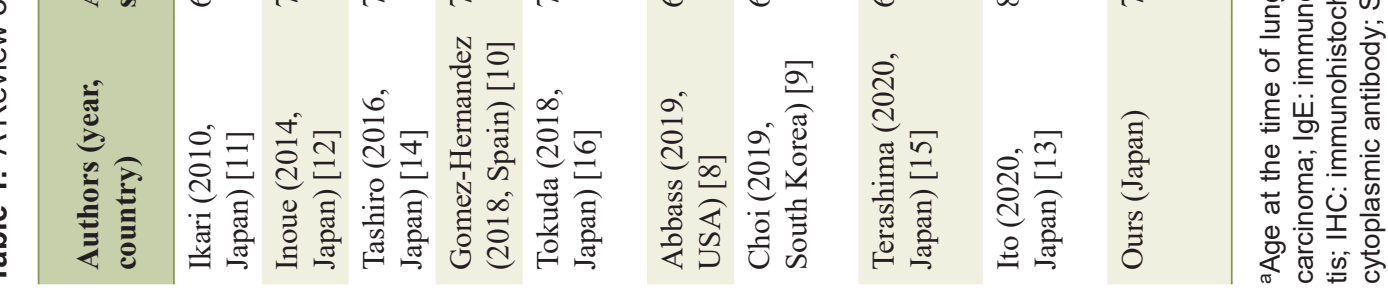


[6]. On the other hand, according to two Japanese nationwide surveys of 744 and 1,763 lung surgeries for lung cancer with idiopathic interstitial pneumonia, the prevalence of acute exacerbation following surgery was $8.3 \%$ (in 62 cases) and $9.3 \%$ (in 164 patients ), and the mortality rate was $3.5 \%$ (in 26 cases) and $2.6 \%$ (in 46 cases), respectively $[22,23]$. Among seven independent risk factors of acute exacerbation identified in the previous study [23], our case met at least four risk factors: surgical procedure of lobectomy, male gender, history of exacerbation and preoperative steroid use. We could not access percent vital capacity preoperatively. Thus, in terms of interstitial lung diseases, post-surgical acute exacerbation might be very likely in our case. There was no report of post-operative acute exacerbation and mortality in IgG4-related lung disease. Our case, in which acute exacerbation occurred soon after lung surgery, calls physicians' attention to a risk of post-operative acute exacerbation.

\section{Conclusion}

In conclusion, our case alerts oncologists to IgG4-related disease as a possible underlying comorbidity which confuses pretreatment clinical staging and treatment strategy.

\section{Acknowledgments}

None to declare.

\section{Financial Disclosure}

None to declare.

\section{Conflict of Interest}

None to declare.

\section{Informed Consent}

Not applicable because the manuscript has been sufficiently de-identified to protect the patient.

\section{Author Contributions}

S. Ikebe, S. Minami, S. Ihara and K. Komuta were involved in treatment of this patients. H. Yasuoka performed the pathological diagnosis. S. Ikebe drafted the report. All authors read and critically reviewed the report. All authors approved the final submitted version.

\section{Data Availability}

The authors declare that data supporting the findings of this study are available within the article.

\section{References}

1. Ahn SS, Song JJ, Park YB, Lee SW. Malignancies in Korean patients with immunoglobulin G4-related disease. Int J Rheum Dis. 2017;20(8):1028-1035.

2. Yamamoto M, Takahashi H, Tabeya T, Suzuki C, Naishiro $\mathrm{Y}$, Ishigami K, Yajima $\mathrm{H}$, et al. Risk of malignancies in IgG4-related disease. Mod Rheumatol. 2012;22(3):414418.

3. Gupta R, Khosroshahi A, Shinagare S, Fernandez C, Ferrone C, Lauwers GY, Stone JH, et al. Does autoimmune pancreatitis increase the risk of pancreatic carcinoma?: a retrospective analysis of pancreatic resections. Pancreas. 2013;42(3):506-510.

4. Shiokawa M, Kodama Y, Yoshimura K, Kawanami C, Mimura J, Yamashita Y, Asada M, et al. Risk of cancer in patients with autoimmune pancreatitis. Am J Gastroenterol. 2013;108(4):610-617.

5. Hirano K, Tada M, Sasahira N, Isayama H, Mizuno S, Takagi $\mathrm{K}$, Watanabe $\mathrm{T}$, et al. Incidence of malignancies in patients with IgG4-related disease. Intern Med. 2014;53(3):171-176.

6. Corcoran JP, Culver EL, Anstey RM, Talwar A, Manganis CD, Cargill TN, Hallifax RJ, et al. Thoracic involvement in IgG4-related disease in a UK-based patient cohort. Respir Med. 2017;132:117-121.

7. Inoue D, Zen Y, Abo H, Gabata T, Demachi H, Kobayashi $\mathrm{T}$, Yoshikawa $\mathrm{J}$, et al. Immunoglobulin G4-related lung disease: CT findings with pathologic correlations. Radiology. 2009;251(1):260-270.

8. Abbass K, Krug H. Granulomatosis with polyangiitis in a patient with biopsy-proven IgG4-related pulmonary disease and coincident small cell lung cancer. BMJ Case Rep. 2019;12(3):e226280.

9. Choi S, Park S, Chung MP, Kim TS, Cho JH, Han J. A rare case of adenosquamous carcinoma arising in the background of IgG4-related lung disease. J Pathol Transl Med. 2019;53(3):188-191.

10. Gomez Hernandez MT, Rodriguez Alvarado I, Novoa N, Jimenez Lopez MF. Immunoglobulin G4-Related Lung Disease as an Incidental Finding After Surgical Resection of Lung Cancer. Arch Bronconeumol. 2019;55(5):276278.

11. Ikari J, Kojima M, Tomita K, Nakamura T, Toyoda F, Otsuki Y, Shimizu S, et al. A case of IgG4-related lung disease associated with multicentric Castleman's disease and lung cancer. Intern Med. 2010;49(13):1287-1291.

12. Inoue T, Hayama M, Kobayashi S, Oyaizu T, Nakazato Y, Honma K, Chida M. Lung cancer complicated with IgG4related disease of the lung. Ann Thorac Cardiovasc Surg. 2014;20(Suppl):474-477.

13. Ito Y, Harada M, Kagoo N, Kubota T, Ichijyo K, Mochizuki E, Uehara M, et al. Axillary lymphadenopathy with IgG4 positive plasma cell infiltration as differential diagnosis of metastatic lung adenocarcinoma. Respir Med Case Rep. 2020;31:101196. 
14. Tashiro H, Takahashi K, Nakamura T, Komiya K, Kimura S, Sueoka-Aragane N. Coexistence of lung cancer and immunoglobulin G4-related lung disease in a nodule: a case report. J Med Case Rep. 2016;10(1):113.

15. Terashima T, Iwami E, Shimada T, Kuroda A, Matsuzaki T, Nakajima T, Sasaki A, et al. IgG4-related pleural disease in a patient with pulmonary adenocarcinoma under durvalumab treatment: a case report. BMC Pulm Med. 2020;20(1):104.

16. Tokuda K, Nakaya T, Nakayama M, Bando M, Sugiyama Y, Hagiwara H. Primary lung adenocarcinoma associated with IgG4-related lung disease. Annals of the Japanese Respiratory Society. 2018;7(1):20-24.

17. Umehara H, Okazaki K, Masaki Y, Kawano M, Yamamoto $\mathrm{M}$, Saeki T, Matsui S, et al. A novel clinical entity, IgG4-related disease (IgG4RD): general concept and details. Mod Rheumatol. 2012;22(1):1-14.

18. Wallace ZS, Naden RP, Chari S, Choi HK, Della-Torre E, Dicaire JF, Hart PA, et al. The 2019 American College of Rheumatology/European League Against Rheumatism classification criteria for IgG4-related disease. Ann
Rheum Dis. 2020;79(1):77-87.

19. Matsui S, Yamamoto H, Minamoto S, Waseda Y, Mishima M, Kubo K. Proposed diagnostic criteria for IgG4-related respiratory disease. Respir Investig. 2016;54(2):130132.

20. Wang Y, Zhu J. Need for caution in diagnosing IgG4-Related disease: a possible paraneoplastic syndrome? Comment on the Article by Wallace et al. Arthritis Rheumatol. 2017;69(3):681-682.

21. Haider M, Haji F, Alalwan O, Aljufairi E, Shah TS. IgG4Related Disease, the Malignancy Mimicker: Case Series from Bahrain. Case Rep Rheumatol. 2018;2018:4057024.

22. Miyamoto A, Kishi K, Yoshimura K. [A nationwide survey concerning lung surgery for lung cancer associated with idiopathic interstitial pneumonia]. Nihon Kokyuki Gakkai Zasshi. 2011;49(2):148-150.

23. Sato T, Teramukai S, Kondo H, Watanabe A, Ebina M, Kishi K, Fujii Y, et al. Impact and predictors of acute exacerbation of interstitial lung diseases after pulmonary resection for lung cancer. J Thorac Cardiovasc Surg. 2014;147(5):1604-1611 e11603. 